\title{
Group classification of $(1+1)$-Dimensional Schrödinger Equations with Potentials and Power Nonlinearities
}

\author{
Roman O. POPOVYCH ${ }^{\dagger}$, Nataliya M. IVANOVA ${ }^{\ddagger}$ and Homayoon ESHRAGHI * \\ ${ }_{\dagger, \ddagger}$ Institute of Mathematics of NAS of Ukraine, 3 Tereshchenkivska Str., 01601 Kyiv-4, Ukraine \\ E-mail: †rop@imath.kiev.ua, ${ }^{\dagger}$ ivanova@imath.kiev.ua \\ * Institute for Studies in Theor. Physics and Mathematics, Tehran P.O. Box: 19395-5531, Iran \\ E-mail: eshraghi@theory.ipm.ac.ir
}

\begin{abstract}
We perform the complete group classification in the class of nonlinear Schrödinger equations of the form $i \psi_{t}+\psi_{x x}+|\psi|^{\gamma} \psi+V(t, x) \psi=0$ where $V$ is an arbitrary complex-valued potential depending on $t$ and $x, \gamma$ is a real non-zero constant. We construct all the possible inequivalent potentials for which these equations have non-trivial Lie symmetries using a combination of algebraic and compatibility methods. The proposed approach can be applied to solving group classification problems for a number of important classes of differential equations arising in mathematical physics.
\end{abstract}

\section{Introduction}

Nonlinear Schrödinger equations (NSchEs) are important objects for investigation in different fields of physics and mathematics. They are used in geometric optics [6], nonlinear quantum mechanics [4] and the theory of Bose-Einstein condensation. NSchEs also have a number of applications in wave propagation in inhomogeneous medium and arise as a model of plasma phenomena. The cubic Schrödinger equation is one of the most known integrable models of mathematical physics. At the same time the physical interpretation of some known types of nonlinear Schrödinger equations is not completely clear and is an interesting problem to solve.

Schrödinger equations have been investigated by means of symmetry methods by a number of authors. (See e.g. [17, 12, 18, 15, 7, 8, 9, 10, 11, 13] and references therein for classical Lie symmetries.) In fact, group classification for Schrödinger equations was first performed by S. Lie. More precisely, his classification [14] of all the linear equations with two independent complex variables contains, in an implicit form, solving the classification problem for the linear $(1+1)$-dimensional Schrödinger equations with an arbitrary potential. And it is follows from Lie's proof that the equations for the harmonic and repulsive oscillators and free fall are locally equivalent to the free Schrödinger equation.

To the best of our knowledge, actual investigations of Lie symmetries for Schrödinger equations were started in 1970s with the linear case $[17,18,15]$. The next considered class covered $(1+n)$-dimensional NSchEs with nonlinearities of the form $f(|\psi|) \psi$, which are notable for their symmetry properties because any such equation is invariant with respect to the Galilean group. It turned out that extensions of this invariance group are possible only for the logarithm and power functions, and there exists the power value $\gamma=4 / n$ which is special with respect to the symmetry point of view [7]. Namely, the free Schrödinger equation and the NSchE with the nonlinearity $|\psi|^{4 / n} \psi$ are distinctive ones from a lot of similar equations, since they admit the complete Galilei group extended with both the scale and conformal transformations. (Here $n$ is the number of spatial variables, and for $n=1$ and $n=2$ they are the quintic and cubic 
equations respectively that stand out against the other NSchEs.) This NSchE has also other special properties, and the value $\gamma=4 / n$ is called now the critical power.

The results mentioned above formed a basis for symmetry studying more extended classes of NSchEs. So, finishing the series of papers $[8,9,10,11,12]$ on group analysis and exact solutions of NSchEs, L. Gagnon and P. Winternitz [12] investigated a general class of (1+1)-dimensional variable coefficient cubic SchEs. It is the symmetry approach that was applied by H.-D. Doebner and G.A. Goldin to obtain new equations which generalize the Schrödinger equation and can be used in nonlinear quantum mechanics [4]. These equations were investigated in more detail with the symmetry point of view by a number of authors $[16,5,26]$. The complete group classification of constant coefficient NSchEs with nonlinearities of the general form $F=F\left(\psi, \psi^{*}\right)$ was performed by A.G. Nikitin and R.O. Popovych [19].

Group classification is one of symmetry methods used to choose physically relevant models from parametric classes of systems of (partial or ordinary) differential equations. The parameters can be constants or functions of independent variables, unknown functions and their derivatives. Exhaustive consideration of the problem of group classification for a parametric class $\mathcal{L}$ of systems of differential equations includes the following steps:

1. Finding the group $G^{\mathrm{ker}}$ (the kernel of maximal invariance groups) of local transformations that are symmetries for all systems from $\mathcal{L}$.

2. Construction of the group $G^{\text {equiv }}$ (the equivalence group) of local transformations which transform $\mathcal{L}$ into itself.

3. Description of all possible $G^{\text {equiv }}$-inequivalent values of parameters that admit maximal invariance groups wider than $G^{\mathrm{ker}}$.

Following S. Lie, one usually considers infinitesimal transformations instead of finite ones. This approach essentially simplifies the problem of group classification, reducing it to problems for Lie algebras of vector fields. See $[26,19,25,21,24,22,1,2]$ for precise formulation of group classification problems and more details on the used methods.

In this paper we study a class of NSchEs of the form

$$
i \psi_{t}+\psi_{x x}+|\psi|^{\gamma} \psi+V \psi=0,
$$

where the potential $V=V(t, x)$ is an arbitrary complex-valued smooth function of the variables $t$ and $x, \gamma$ is a real non-zero constant. (Here and below subscripts of functions denote differentiation with respect to the respective variables.) To find a complete set of inequivalent cases of $V$ admitting extensions of the maximal Lie invariance algebra, we combine the classical Lie approach, studying the algebra generated by all the possible Lie symmetry operators for equations from class (1) (the adjoint representation, the inequivalent one-dimensional subalgebras etc.) and investigation of compatibility of classifying equations. The subclass of (1) where $\gamma=2$ (the cubic SchEs with potentials) has been investigated in [23] in a similar way.

In fact, here we solve three classification problems for different classes of equations having the form (1): with the potentials depending only on $t$ (Section III), with the stationary potentials (Section IV) and the general case with arbitrary potentials (Section II). Moreover, it is proved in Section II the constant $\gamma$ can be assumed as fixed under our consideration. And there exists a special constant $\widehat{\gamma}$ depending on the power $\gamma\left(\widehat{\gamma}=\gamma^{-1}(4-\gamma)\right)$, which arises at the beginning of the classification procedure when classification condition (3) is constructed and explicitly appears in the final results of classification. The value $\gamma=4$ (quintic nonlinearity, it is the same that $\widehat{\gamma}=0$ ) is special with respect to group classification in class (1). 
The classification approach used in this paper allows us to formulate a necessary and sufficient condition of mutual equivalence for the cases of extensions of maximal invariance algebras in algebraic terms (Corollary 2). The classical stationary potentials (free particle, the harmonic and repulsive oscillators, free fall, radial free particle, the radial harmonic and repulsive oscillators [15]) naturally arise under the group classification with respect to the (smaller) equivalence group in the class of stationary potentials. Using Corollary 2 and the complete equivalence group of class (1) we easily construct transformations of these $x$-dependent potentials to $x$-free ones in explicit form (see Remark 8).

\section{General Case $V=V(t, x)$}

Consider an operator $Q=\xi^{t} \partial_{t}+\xi^{x} \partial_{x}+\eta \partial_{\psi}+\eta^{*} \partial_{\psi^{*}}$ from the maximal Lie invariance algebra $A^{\max }(\gamma, V)$ of equation (1) with a power $\gamma$ and a potential $V$. Here $\xi^{t}, \xi^{x}$ and $\eta$ are smooth functions of $t, x, \psi$ and $\psi^{*}$. The infinitesimal invariance condition [21,20] of equation (1) with respect to the operator $Q$ implies the linear overdetermined system on the coefficients of $Q$ :

$$
\begin{aligned}
& \xi_{\psi}^{t}=\xi_{\psi^{*}}^{t}=\xi_{x}^{t}=0, \quad \xi_{\psi}^{x}=\xi_{\psi^{*}}^{x}=0, \quad \xi_{t}^{t}=2 \xi_{x}^{x}, \quad \eta_{\psi^{*}}=\eta_{\psi \psi}=0, \quad \psi \eta_{\psi}=\eta, \\
& 2 \eta_{\psi x}=i \xi_{t}^{x}, \quad \gamma\left(\eta_{\psi}+\eta_{\psi}^{*}\right)=-2 \xi_{t}^{t}, \quad i \eta_{\psi t}+\eta_{\psi x x}+\xi^{t} V_{t}+\xi^{x} V_{x}+\xi_{t}^{t} V=0 .
\end{aligned}
$$

Therefore, the following theorem holds.

Theorem 1. Any operator $Q$ from $A^{\max }(\gamma, V)$ of equation (1) with arbitrary potential $V$ lies in the linear span of operators of the form

$$
D(\xi)=\xi \partial_{t}+\frac{1}{2} \xi_{t} x \partial_{x}+\frac{1}{8} \xi_{t t} x^{2} M-\frac{1}{\gamma} \xi_{t} I, \quad G(\chi)=\chi \partial_{x}+\frac{1}{2} \chi_{t} x M, \quad \lambda M .
$$

Here $\chi=\chi(t), \xi=\xi(t)$ and $\lambda=\lambda(t)$ are arbitrary smooth functions of $t, M=i\left(\psi \partial_{\psi}-\psi^{*} \partial_{\psi^{*}}\right)$, $I=\psi \partial_{\psi}+\psi^{*} \partial_{\psi^{*}}$. Moreover, the coefficients of $Q=D(\xi)+G(\chi)+\lambda M \in A^{\max }(\gamma, V)$ should satisfy the classifying condition

$$
\xi V_{t}+\left(\frac{1}{2} \xi_{t} x+\chi\right) V_{x}+\xi_{t} V=\frac{1}{8} \xi_{t t t} x^{2}+\frac{1}{2} \chi_{t t} x+\lambda_{t}+i \frac{\widehat{\gamma}}{4} \xi_{t t}
$$

Here and below $\widehat{\gamma}=\gamma^{-1}(4-\gamma)$.

Theorem 2. The Lie algebra of the kernel of maximal Lie invariance groups of equations from class (1) is $A^{\mathrm{ker}}=\langle M\rangle$.

Note 1. Sometimes (e.g. for reduction and construction of solutions) it is convenient to use the amplitude $\rho$ and the phase $\varphi$ instead of the wave function $\psi=\rho e^{i \varphi}$. Then equation (1) is replaced by the system for two real-valued functions $\rho$ and $\varphi$ :

$$
\rho_{t}+2 \rho_{x} \varphi_{x}+\rho \varphi_{x x}+\rho \operatorname{Im} V=0, \quad-\rho \varphi_{t}-\rho\left(\varphi_{x}\right)^{2}+\rho_{x x}+\rho^{\gamma+1}+\rho \operatorname{Re} V=0 .
$$

Operators (2) have the same form with $M=\partial_{\varphi}, I=\rho \partial_{\rho}$.

To study equivalence transformations for class (1), both the infinitesimal and direct method are used. In the framework of the infinitesimal method we consider a first-order differential operator of the most general form in the space of the variables $t, x, \psi, \psi^{*}, V, V^{*}$ and $\gamma$, i.e.

$$
Q=\xi^{t} \partial_{t}+\xi^{x} \partial_{x}+\eta \partial_{\psi}+\eta^{*} \partial_{\psi^{*}}+\theta \partial_{V}+\theta^{*} \partial_{V^{*}}+\Gamma \partial_{\gamma}
$$


where $\xi^{t}, \xi^{x}, \eta, \theta$ and $\Gamma$ may depend on all the variables, and assume it being an infinitesimal symmetry operator for the system

$$
i \psi_{t}+\psi_{x x}+|\psi|^{\gamma} \psi+V \psi=0, \quad \gamma_{t}=\gamma_{x}=\gamma_{\psi}=\gamma_{\psi^{*}}=0, \quad V_{\psi}=V_{\psi^{*}}=0 .
$$

(Under the prolongation procedure for equivalence transformations we suppose $\psi$ is a function of $t$ and $x$ as well as $V$ and $\gamma$ are functions of $t, x$ and $\psi$.)

Theorem 3. The Lie algebra $A^{\text {equiv }}$ of the equivalence group $G^{\text {equiv }}$ of class (1) is generated by the operators

$$
\begin{aligned}
& D^{\prime}(\xi)=D(\xi)+\frac{1}{8} \xi_{t t t} x^{2}\left(\partial_{V}+\partial_{V^{*}}\right)+\frac{i}{\gamma} \xi_{t t}\left(\partial_{V}-\partial_{V^{*}}\right)-\xi_{t}\left(V \partial_{V}+V^{*} \partial_{V^{*}}\right), \\
& G^{\prime}(\chi)=G(\chi)+\frac{1}{2} \chi_{t t} x\left(\partial_{V}+\partial_{V^{*}}\right), \quad M^{\prime}(\lambda)=\lambda M+\lambda_{t}\left(\partial_{V}+\partial_{V^{*}}\right) .
\end{aligned}
$$

In the framework of the direct method we look for all local transformations in the space of the variables $t, x, \psi, \psi^{*}, V, V^{*}$ and $\gamma$, which preserve system (4).

Theorem 4. The equivalence group $G^{\text {equiv }}$ of the class (1) is generated by the family of continuous transformations

$$
\begin{aligned}
& \tilde{t}=T, \quad \tilde{x}=x \sqrt{T_{t}}+X, \quad \tilde{\psi}=\psi \frac{1}{\sqrt{T_{t}}} \exp \left(\frac{i}{8} \frac{T_{t t}}{T_{t}} x^{2}+\frac{i}{2} \frac{X_{t}}{\sqrt{T_{t}}} x+i \Psi\right), \quad \tilde{\gamma}=\gamma, \\
& \tilde{V}=\frac{1}{T_{t}}\left(V+\frac{1}{8}\left(\frac{T_{t t}}{T_{t}}\right)_{t} x^{2}+\frac{1}{2}\left(\frac{X_{t}}{\sqrt{T_{t}}}\right)_{t} x+i \frac{\widehat{\gamma}}{4} \frac{T_{t t}}{T_{t}}-\left(\frac{1}{4} \frac{T_{t t}}{T_{t}} x+\frac{1}{2} \frac{X_{t}}{\sqrt{T_{t}}}\right)^{2}+\Psi_{t}\right)
\end{aligned}
$$

and two discrete transformations: the space reflection $I_{x}(\tilde{t}=t, \tilde{x}=-x, \tilde{\psi}=\psi, \tilde{\gamma}=\gamma, \tilde{V}=V)$ and the Wigner time reflection $I_{t}\left(\tilde{t}=-t, \tilde{x}=x, \tilde{\psi}=\psi^{*}, \tilde{\gamma}=\gamma, \tilde{V}=V^{*}\right)$. Here $T, X$ and $\Psi$ are arbitrary smooth functions of $t, T_{t}>0$.

We also prove the stronger statement than Theorem 4.

Theorem 5. If two equations from class (1) with the parameter values $(\gamma, V)$ and $(\tilde{\gamma}, \tilde{V})$ are transformed each to other by local transformations then $\tilde{\gamma}=\gamma$. Moreover, since $\gamma \neq 0$ any transformation of such type belongs to $G^{\text {equiv }}$.

Note 2. It follows from Theorems 4 and 5 that there exist no equivalence and, moreover, local transformations changing $\gamma$. Therefore, we can assume that $\gamma$ is fixed in our consideration below and omit it from notations of the maximal Lie invariance algebras of an equation of form (1) etc.

Note 3. The linear span of operators of the form (2) ( $\gamma$ is fixed!) is an (infinite-dimensional) Lie algebra $A^{\cup}$ under the usual Lie bracket of vector fields. Since for any $Q \in A^{\cup}$ where $\left(\xi^{t}, \xi^{x}\right) \neq(0,0)$ we can find $V$ satisfying condition (3) then $A^{\cup}=\left\langle\bigcup_{V} A^{\max }(V)\right\rangle$. The non-zero commutation relations between the basis elements of $A^{\cup}$ are the following ones:

$$
\begin{aligned}
& {\left[D\left(\xi^{1}\right), D\left(\xi^{2}\right)\right]=D\left(\xi^{1} \xi_{t}^{2}-\xi^{2} \xi_{t}^{1}\right), \quad[D(\xi), G(\chi)]=G\left(\xi \chi_{t}-\frac{1}{2} \xi_{t} \chi\right), \quad[D(\xi), \lambda M]=\xi \lambda_{t} M,} \\
& {\left[G\left(\chi^{1}\right), G\left(\chi^{1}\right)\right]=\frac{1}{2}\left(\chi^{1} \chi_{t}^{2}-\chi^{2} \chi_{t}^{1}\right) M .}
\end{aligned}
$$


We use the notation $\operatorname{Aut}\left(A^{\cup}\right)$ for the automorphism group acting on $A^{\cup}$, which is generated by all the one-parameter groups corresponding to the adjoint representations of operators of $A^{\cup}$ into $A^{\cup}$ and two discrete transformations $\operatorname{Ad} I_{x}$ and $\operatorname{Ad} I_{t}$ included additionally. The actions of $\operatorname{Ad} I_{x}$ and Ad $I_{t}$ on the basis elements of $A^{\cup}$ are defined by the formulas $\operatorname{Ad} I_{x} G(\chi)=G(-\chi)$ (the other basis operators do not change) and $\operatorname{Ad} I_{t} D(\xi)=D(\tilde{\xi})$, Ad $I_{t} G(\chi)=G(\tilde{\chi})$, Ad $I_{t} \lambda M=\tilde{\lambda} M$, where $\tilde{\xi}(t)=-\xi(-t), \tilde{\chi}(t)=\chi(-t)$ and $\tilde{\lambda}(t)=-\lambda(-t)$.

Corollary 1. $A^{\text {equiv }} \simeq A^{\cup}, G^{\text {equiv }} \simeq \operatorname{Aut}\left(A^{\cup}\right)$, and the isomorphism is determined by means of prolongation of operators from $A^{\cup}$ to the space $\left(\gamma, V, V^{*}\right)$.

Corollary 2. Let $A^{1}$ and $A^{2}$ be the maximal Lie invariance algebras of equations from class (1) for some potentials, and $\mathcal{V}^{i}=\left\{V \mid A^{\max }(V)=A^{i}\right\}, i=1,2$. Then $\mathcal{V}^{1} \sim \mathcal{V}^{2} \bmod G^{\text {equiv }}$ iff $A^{1} \sim A^{2} \bmod \operatorname{Aut}\left(A^{\cup}\right)$.

Lemma 1. A complete list of Aut $A^{\cup}$-inequivalent one-dimensional subalgebras of $A^{\cup}$ is exhausted by the algebras $\left\langle\partial_{t}\right\rangle,\left\langle\partial_{x}\right\rangle,\langle t M\rangle,\langle M\rangle$.

Proof: Consider any operator $Q \in A^{\cup}$, i.e. $Q=D(\xi)+G(\chi)+\lambda M$. Depending on the values of $\xi, \chi$ and $\lambda$ it is equivalent under $\operatorname{Aut}\left(A^{\cup}\right)$ and multiplication by a number to one from the following operators: $D(1)$ if $\xi \neq 0 ; G(1)$ if $\xi=0$ and $\chi \neq 0 ; t M$ if $\xi=\chi=0, \lambda_{t} \neq 0 ; \quad M$ if $\xi=\chi=\lambda_{t}=0$.

Corollary 3. If $A^{\max }(V) \neq A^{\text {ker }}$ then $V_{t} V_{x}=0 \bmod G$ equiv .

Proof: Under the corollary assumption there exists an operator $Q=D(\xi)+G(\chi)+\lambda M \in$ $A^{\max }(V)$ which do not belong to $\langle M\rangle$. Condition (3) implies $(\xi, \chi) \neq(0,0)$. Therefore, in force of Lemma $1\langle Q\rangle \sim\left\langle\partial_{t}\right\rangle$ or $\left\langle\partial_{x}\right\rangle \bmod$ Aut $A^{\cup}$, i.e. $V_{t} V_{x}=0 \bmod G^{\text {equiv }}$.

Theorem 6. A complete set of inequivalent cases of $V$ admitting extensions of the maximal Lie invariance algebra of equations (1) is exhausted by the potentials given in Table 1.

Table 1. Results of classification. Here $W(t), \nu, \alpha, \beta \in \mathbb{R},(\alpha, \beta) \neq(0,0)$.

\begin{tabular}{|r|c|l|}
\hline $\mathrm{N}$ & $V$ & \multicolumn{1}{|c|}{ Basis of $A^{\max }$} \\
\hline 0 & $V(t, x)$ & $M$ \\
\hline 1 & $i W(t)$ & $M, \partial_{x}, G(t)$ \\
2 & $\frac{i}{2} \frac{\widehat{\gamma} t+\nu}{t^{2}+1}$ & $M, \partial_{x}, G(t), D\left(t^{2}+1\right)$ \\
3 & $i \nu t^{-1}, \quad \nu \neq 0, \frac{\widehat{\gamma}}{2}$ & $M, \partial_{x}, G(t), D(t)$ \\
4 & $i$ & $M, \partial_{x}, G(t), \partial_{t}$ \\
5 & $0, \gamma \neq 4$ & $M, \partial_{x}, G(t), \partial_{t}, D(t)$ \\
& $\gamma=4$ & $M, \partial_{x}, G(t), \partial_{t}, D(t), D\left(t^{2}\right)$ \\
6 & $V(x)$ & $M, \partial_{t}$ \\
7 & $(\alpha+i \beta) x^{-2}, \gamma \neq 4$ & $M, \partial_{t}, D(t)$ \\
& \multicolumn{2}{|l}{} \\
& \multicolumn{2}{|l}{} \\
\hline
\end{tabular}

For convenience we use below the double numeration T.N of classification cases where $\mathrm{T}$ is a table number and $\mathrm{N}$ is a row number. 
Note 4. We mean that the invariance algebras for Cases 1.0, 1.1, 1.6 and analogous ones from Tables 2 and 3 are maximal if these cases are inequivalent under the corresponding equivalence group to the other, more specialized, cases from the same table.

Note 5. There exists a discrete equivalence transformation $\tau$ for the set of potentials $i \nu t^{-1}$, $\nu \in \mathbb{R}$, which has form (5) with $T=-t^{-1}, X=0, \Psi=0$. It transforms $\nu$ in the following way: $\nu \rightarrow \widehat{\gamma} / 2-\nu$. For the cases under consideration to be completely inequivalent, we have to assume additionally that $\nu \geq \widehat{\gamma} / 4$ (or $\nu \leq \widehat{\gamma} / 4$ ) in Case 1.3. Since $I_{t} \in G$ equiv we can assume analogously $\nu \geq 0$ in Case 1.2 and $\beta \geq 0$ in Case 1.7. Moreover, $\tau$ is a discrete symmetry transformation for Case $1.3(\nu=\widehat{\gamma} / 4)$ and, as a limit of the continuous transformations generated by the operator $D\left(t^{2}+1\right)$, for Case 1.2 .

If we use Corollary 3 then to prove Theorem 6 it is sufficient to study two cases: $V_{x}=0$ and $V_{t}=0$. In fact, below we obtain the complete results of group classifications for both special cases and then unite them for the general case under consideration.

\section{Case $V=V(t)$}

Consider the equations from class (1) with potentials satisfying the additional assumption $V_{x}=$ 0, i.e. $V=V(t)$. The following chain of lemmas gives complete solving of classification problem in this subclass.

Lemma 2. $A_{V_{x}=0}^{\mathrm{ker}}=\langle M, G(1), G(t)\rangle$.

Lemma 3. $A_{V_{x}=0}^{\text {equiv }}=\left\langle M^{\prime}(\lambda) \forall \lambda=\lambda(t), G^{\prime}(1), G^{\prime}(t), D^{\prime}(1), D^{\prime}(t), D^{\prime}\left(t^{2}\right)\right\rangle$. $G_{V_{x}=0}^{\text {equiv }}$ is generated by $I_{t}, I_{x}$ and the transformations of form (5), where $T=\left(a_{1} t+a_{0}\right) /\left(b_{1} t+b_{0}\right), X=c_{1} t+c_{0}, \Psi$ is an arbitrary smooth function of $t . a_{i}, b_{i}$ and $c_{i}$ are arbitrary constants such that $a_{1} b_{0}-b_{1} a_{0}>0$.

Lemma 4. For any $V=V(t): V \sim i W \bmod G_{V_{x}=0}^{\text {equiv }}$ where $W=\operatorname{Im} V$, i.e. $W=W(t) \in \mathbb{R}$.

Lemma 5. $A_{\{i W\}}^{\mathrm{ker}}=A_{V_{x}=0}^{\mathrm{ker}} . A^{\max }(i W) \subset A_{\{i W\}}^{\cup}=A_{\{i W\}}^{\mathrm{ker}} \boxplus S$ where $S=\left\langle D(1), D(t), D\left(t^{2}\right)\right\rangle$. $A_{\{i W\}}^{\cup}=\bigcup_{W} A^{\max }(i W) \cdot A_{\{i W\}}^{\text {equiv }}=\left\langle M, G^{\prime}(1), G^{\prime}(t), D^{\prime}(1), D^{\prime}(t), D^{\prime}\left(t^{2}\right)\right\rangle . G_{\{i W\}}^{\text {equiv }}=\left.G_{V_{x}=0}^{\text {equiv }}\right|_{\Psi=\text { const }}$. $A_{\{i W\}}^{\cup} \simeq A_{\{i W\}}^{\text {equiv }}=\operatorname{pr}_{\left(V, V^{*}\right)} A_{\{i W\}}^{\cup}$.

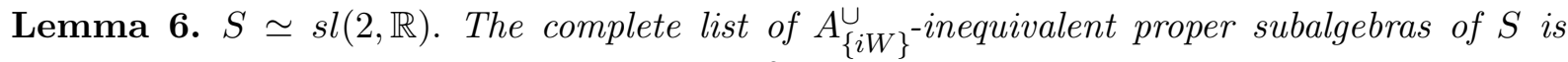
exhausted by the algebras $\langle D(1)\rangle,\langle D(t)\rangle,\left\langle D\left(t^{2}+1\right)\right\rangle,\langle D(1), D(t)\rangle$.

Lemma 7. Let $A^{1}$ and $A^{2}$ be the maximal Lie invariance algebras of equations from class (1) for some potentials from $\{i W(t)\}$, and $\mathcal{W}^{i}=\left\{W(t) \mid A^{\max }(i W)=A^{i}\right\}, i=1,2$. Then $\mathcal{W}^{1} \sim \mathcal{W}^{2}$ $\bmod G_{\{i W\}}^{\text {equiv }}$ iff $A^{1} \cap S \sim A^{2} \bigcap S \bmod \operatorname{Aut}(S)$.

Lemma 8. If $A_{\{i W\}}^{\max } \neq A_{V_{x}=0}^{\mathrm{ker}}$ the potential $i W(t)$ is $G_{\{i W\}}^{\text {equiv }}$-equivalent to one from Cases 1.2-1.5.

Note 6. If $\gamma \neq 4$ or $W \neq$ const $A^{\max }(i W) \not \supset S$ (otherwise, condition (3) would imply an incompatible system for $W$ ). If $W=$ const $W \in\{0,1\} \bmod G_{\{i W\}}^{\text {equiv }}$ (Cases 1.5 and 1.4 correspondingly). Cases $1.2 \nu$ and $1.2 \tilde{\nu}\left(1.3 \nu\right.$ and $1.3 \tilde{\nu}$ where $\left.\nu, \tilde{\nu} \geq \frac{1}{4}\right)$ are $G^{\text {equiv }_{\text {-inequivalent if }}}$ $\nu \neq \tilde{\nu}$. Since $D\left(t^{2}+1\right)$ cannot be contained in any two-dimensional subalgebra of $S$ it is not possible to extend $A^{\max }$ in Case 1.2. There are two possibilities for extension of $A^{\max }\left(i \nu t^{-1}\right)$, namely with either $D(1)$ (for $\nu=0$, Case 1.5) or $D\left(t^{2}\right)$ (for $\nu=(4-\gamma) /(2 \gamma)$ that is equivalent to Case 1.5 with respect to $\left.G_{\{i W\}}^{\text {equiv }}\right)$. That is why for $\nu=0, \gamma=4$ dimension of $A^{\text {max }}$ is greatest. 


\section{Case $\boldsymbol{V}=\boldsymbol{V}(\boldsymbol{x})$}

Consider class (1) with the additional assumption $V_{t}=0$, i.e. $V=V(x)$.

Lemma 9. $A_{V_{t}=0}^{\mathrm{ker}}=\langle M, D(1)\rangle$.

Lemma 10. $A_{V_{t}=0}^{\text {equiv }}=\left\langle M^{\prime}(1), M^{\prime}(t), G^{\prime}(1), D^{\prime}(1), D^{\prime}(t)\right\rangle . G_{V_{t}=0}^{\text {equiv }}$ is generated by $I_{t}, I_{x}$ and the transformations of form (5) where $T_{t t}=X_{t}=\Psi_{t t}=0$.

Lemma 11. If $A^{\max }(V) \neq A_{V_{t}=0}^{\mathrm{ker}}$ the potential $V(x)$ is $G_{V_{t}=0}^{\text {equiv }}$-equivalent to one from cases of either Table 2 if $\gamma \neq 4$ or Table 3 if $\gamma=4$. (Since $I_{t} \in G$ equiv we can assume $\nu \geq 0$ in Cases 2.5, 2.6, 3.5-3.7, $\nu>0$ in Case 2.4 and $\beta \geq 0$ in Cases 2.1, 3.1-3.3.)

Table 2. Classification of the subclass $V=V(x)$ if $\gamma \neq 4$.

Here $\nu, \alpha, \beta \in \mathbb{R},(\alpha, \beta) \neq(0,0)$.

\begin{tabular}{|c|c|c|l|}
\hline $\mathrm{N}$ & $\mathrm{N}_{1}$ & $V$ & \multicolumn{1}{|c|}{ Basis of $A^{\max }$} \\
\hline 0 & 6 & $V(x)$ & $M, \partial_{t}$ \\
\hline 1 & 7 & $(\alpha+i \beta) x^{-2}$ & $M, \partial_{t}, D(t)$ \\
2 & 7 & $x^{2}+i \widehat{\gamma}+(\alpha+i \beta) x^{-2}$ & $M, \partial_{t}, D\left(e^{4 t}\right)$ \\
3 & 4 & $i$ & $M, \partial_{t}, \partial_{x}, G(t)$ \\
4 & 4 & $x+i \nu, \nu \neq 0$ & $M, \partial_{t}, \partial_{x}+t M, G(2 t)+t^{2} M$ \\
5 & 2 & $-x^{2}+i \nu$ & $M, \partial_{t}, G(\sin 2 t), G(\cos 2 t)$ \\
6 & 3 & $x^{2}+i \nu, \nu \neq \pm \widehat{\gamma}$ & $M, \partial_{t}, G\left(e^{2 t}\right), G\left(e^{-2 t}\right)$ \\
7 & 5 & 0 & $M, \partial_{t}, \partial_{x}, G(t), D(t)$ \\
8 & 5 & $x$ & $M, \partial_{t}, \partial_{x}+t M, G(2 t)+t^{2} M, D(2 t)+G\left(3 t^{2}\right)+t^{3} M$ \\
9 & 5 & $x^{2}+i \widehat{\gamma}$ & $M, \partial_{t}, G\left(e^{2 t}\right), G\left(e^{-2 t}\right), D\left(e^{4 t}\right)$ \\
\hline
\end{tabular}

Table 3. Classification of the subclass $V=V(x)$ if $\gamma=4$.

Here $\nu, \alpha, \beta \in \mathbb{R}, \nu \neq 0,(\alpha, \beta) \neq(0,0)$.

\begin{tabular}{|c|c|c|l|}
\hline $\mathrm{N}$ & $\mathrm{N}_{1}$ & $V$ & \multicolumn{1}{|c|}{ Basis of $A^{\max }$} \\
\hline 0 & 6 & $V(x)$ & $M, \partial_{t}$ \\
\hline 1 & 7 & $(\alpha+i \beta) x^{-2}$ & $M, \partial_{t}, D(t), D\left(t^{2}\right)$ \\
2 & 7 & $x^{2}+(\alpha+i \beta) x^{-2}$ & $M, \partial_{t}, D\left(e^{4 t}\right), D\left(e^{-4 t}\right)$ \\
3 & 7 & $-x^{2}+(\alpha+i \beta) x^{-2}$ & $M, \partial_{t}, D(\cos 4 t), D(\sin 4 t)$ \\
4 & 4 & $i$ & $M, \partial_{t}, \partial_{x}, G(t)$ \\
5 & 4 & $x+i \nu$ & $M, \partial_{t}, \partial_{x}+t M, G(2 t)+t^{2} M$ \\
6 & 2 & $-x^{2}+i \nu$ & $M, \partial_{t}, G(\sin 2 t), G(\cos 2 t)$ \\
7 & 3 & $x^{2}+i \nu$ & $M, \partial_{t}, G\left(e^{2 t}\right), G\left(e^{-2 t}\right)$ \\
8 & 5 & 0 & $M, \partial_{t}, \partial_{x}, G(t), D(t), D\left(t^{2}\right)$ \\
9 & 5 & $x$ & $M, \partial_{t}, \partial_{x}+t M, G(2 t)+t^{2} M$, \\
& & & $D(2 t)+G\left(3 t^{2}\right)+t^{3} M, D\left(4 t^{2}\right)+G\left(4 t^{3}\right)+t^{4} M$ \\
10 & 5 & $x^{2}$ & $M, \partial_{t}, G\left(e^{2 t}\right), G\left(e^{-2 t}\right), D\left(e^{4 t}\right), D\left(e^{-4 t}\right)$ \\
11 & 5 & $-x^{2}$ & $M, \partial_{t}, G(\cos 2 t), G(\sin 2 t), D(\cos 4 t), D(\sin 4 t)$ \\
\hline
\end{tabular}


Proof: Let $V=V(x)$ and $A^{\max }(V) \neq A_{V_{t}=0}^{\mathrm{ker}}$. Consider an arbitrary operator $Q=D(\xi)+G(\chi)+$ $\lambda M \in A^{\max }(V)$. Under Lemma's assumption, the condition (3) implies a set of equations on $V$ of the general form

$$
(a x+b) V_{x}+2 a V=c_{2} x^{2}+c_{1} x+\tilde{c}_{0}+i c_{0}, \quad \text { where } \quad a, b, c_{2}, c_{1}, \tilde{c}_{0}, c_{0}=\text { const } \in \mathbb{R} .
$$

The exact number $k$ of such equations with the linear independent sets of coefficients can be equal to either 1 or 2 . (The value $k=0$ corresponds to the general case $V_{t}=0$ without any extensions of $A^{\max }$.)

For $k=1(a, b) \neq(0,0)$ and there exist two possibilities $a=0$ and $a \neq 0$. If $a=0$ without loss of generality we can put $b=1$. Then condition (3) results in $\xi_{t}=0, c_{2}=c_{0}=0$, i.e. $V_{x}=c_{1} x+\tilde{c}_{0}$, and then $k=2$ that it is impossible.

Therefore, $a \neq 0$ and we can put $a=1$. $\tilde{c}_{0}, b=0 \bmod G_{V_{t}=0}^{\text {equiv }}$. Condition $(3)$ results in $\chi=0$ (then $\left.c_{1}=0\right), \lambda_{t}=0, \widehat{\gamma} \xi_{t t}=2 c_{0} \xi_{t}$ and $\widehat{\gamma} c_{2}=c_{0}^{2}$. For $\gamma=4 c_{0}=0$ and $c_{2} \in\{-4,0,4\} \bmod G_{V_{t}=0}^{\text {equiv }}$ and these possibilities in the value of $c_{2}$ give Cases 3.1-3.3. If $\gamma \neq 4$ we obtain Cases $2.1\left(c_{0}=0\right)$ and $2.2\left(c_{0} \neq 0\right)$.

The condition $k=2$ results in $V=d_{2} x^{2}+d_{1} x+\tilde{d}_{0}+i d_{0} . \tilde{d}_{0}=0 \bmod G_{V_{t}=0}^{\text {equiv }}$. Considering different possibilities for values of the constants $d_{2}, d_{1}$ and $d_{0}$ and taking into account the value of $\gamma$ (either $\gamma \neq 4$ or $\gamma=4$ ), we obtain all the other classification cases:

$$
\begin{aligned}
& d_{2}=d_{1}=d_{0}=0 \rightarrow 2.7,3.8 ; \quad d_{2}=d_{1}=0, d_{0} \neq 0 \rightarrow 2.3,3.4 ; \\
& d_{2}=d_{0}=0, d_{1} \neq 0 \rightarrow 2.8,3.9 ; \quad d_{2}=0, d_{0}, d_{1} \neq 0 \rightarrow 2.4,3.5 ; \\
& d_{2}<0,\left(d_{0}, \widehat{\gamma}\right) \neq(0,0) \rightarrow 2.5,3.6 ; \quad d_{2}<0, d_{0}=\widehat{\gamma}=0 \rightarrow 3.11 ; \\
& d_{2}>0, \widehat{\gamma}^{2} d_{2} \neq d_{0}^{2} \rightarrow 2.6,3.7 ; \quad d_{2}>0, \widehat{\gamma}^{2} d_{2}=d_{0}^{2} \rightarrow 2.9,3.10 .
\end{aligned}
$$

Note 7. To prove Theorem 6 , it is sufficient to consider only the case $k=1, a \neq 0$ in Lemma 11 since other cases of extensions of $A^{\max }(V)$ with $V=V(x)$ admit operators of the form $G(\chi)+\lambda M$ $(\chi \neq 0)$ and, therefore (by Corollary 2$)$, are equivalent to Cases 1.1-1.5.

Note 8. The number $N_{1}$ for each line of Tables 2 and 3 is equal to the number of the same or equivalent case in Table 1. The corresponding equivalence transformations have the form (5) where the functions $T, X$ and $\Psi$ are as follows:

$$
\begin{aligned}
& 2.2,3.2 \rightarrow 1.7,2.6,3.7 \rightarrow 1.3\left(\tilde{\nu}=\frac{\widehat{\gamma}-\nu}{4}\right), 2.9,3.10 \rightarrow 1.5: \quad T=-e^{-4 t}, X=\Psi=0 ; \\
& 3.3 \rightarrow 1.7, \quad 2.5,3.6 \rightarrow 1.2(\tilde{\nu}=\nu), \quad 3.11 \rightarrow 1.5: \quad T=\tan 2 t, X=\Psi=0 ; \\
& 2.4,3.5 \rightarrow 1.4: \quad T=|\nu| t, X=-\sqrt{|\nu|} t^{2}, \Psi=\frac{t^{3}}{3} ; \\
& 2.8,3.9 \rightarrow 1.5: \quad T=t, X=-t^{2}, \Psi=\frac{t^{3}}{3} .
\end{aligned}
$$

Remark 8 completes the proof of Theorem 6 . 


\section{Conclusion}

The results of group classification obtained in this paper can be extended to a more general class of $(1+n)$-dimensional NSchEs with potentials

$$
i \psi_{t}+\Delta \psi+F\left(\psi, \psi^{*}\right)+V(t, \vec{x}) \psi=0,
$$

where the $F=F\left(\psi, \psi^{*}\right)$ is an arbitrary complex-valued smooth function of the variables $\psi$ and $\psi^{*}$. We have already described all possible inequivalent forms of the parameter-function $F$ (without any restriction on the dimension $n$ ) for which an equation of the form (6) with a some potential $V$ has an extension of the maximal Lie invariance algebra. We believe that the classification method suggested in this paper can be effectively applied to complete the group classification in (6) for the small values of $n$. This method can be also a tool to investigate symmetries of other classes of PDEs, and we will attempt to prove general statements on its applicability.

Another direction for our future research to develop the above results is construction of both invariant and partially invariant exact solutions of equations having the form (1) by means of using found Lie symmetries, and knowledge of explicit forms for equivalence transformations (see Theorem 4 and Remark 8) allows us to reduced consideration of known stationary potentials to simpler $x$-free ones. We also plan to study conditional and generalized symmetries of (1) to find non-Lie exact solutions.

As it was shown by Carles [3], the equivalence transformations (5) also give an easy and effective way to produce new results on existence, uniqueness, estimations, etc. of solutions for some equations (1) by means of using known results on other potentials.

\section{Acknowledgements}

The authors are grateful to Profs. V. Boyko, A. Nikitin, I. Yehorchenko and A. Zhalij for useful discussions and interesting comments. The research of NMI was supported by National Academy of Science of Ukraine in the form of the grant for young scientists. ROP appreciate Prof. F. Ardalan (School of Physics, Institute for Studies in Theoretical Physics and Mathematics, Tehran) for hospitality and support during writing this paper.

\section{References}

[1] Akhatov I.Sh., Gazizov R.K. and Ibragimov N.Kh., "Group classification of equation of nonlinear filtration," Dokl. AN SSSR, 293, 1033-1035 (1987) (in Russian).

[2] Akhatov I.Sh., Gazizov R.K. and Ibragimov N.Kh., "Nonlocal symmetries. A heuristic approach," Translated in J. Soviet Math. 55, 1401-1450 (1991). Itogi Nauki i Tekhniki, Current problems in mathematics. Newest results. 34, 3-83 (Moscow, Akad. Nauk SSSR, Vsesoyuz. Inst. Nauchn. i Tekhn. Inform. 1989) (in Russian)

[3] Carles, R., "Critical nonlinear Schrödinger equations with and without harmonic potential," Math. Models Methods Appl. Sci. 12, 1513-1523 (2002). (cond-mat/0112414).

[4] Doebner, H.-D. and Goldin G.A., "Properties of nonlinear Schrödinger equations associated with diffeomorphism group representations," J. Phys. A.: Math. Gen. 27, 1771-1780 (1994).

[5] Doebner, H.-D., Goldin, G.A. and Nattermann, P., "Gauge transformations in quantum mechanics and the unification of nonlinear Schrödinger equations", J. Math. Phys. 40, 49-63 (1999).

[6] Faddeev, L.D. and Takhtajan, L.A., Hamiltonian methods in the theory of solitons (Translated from Russian by A. G. Reyman [A. G. Rel̆man]. Springer Series in Soviet Mathematics. Springer-Verlag, Berlin, 1987). 
[7] Fushchych, W.I. and Moskaliuk, S.S., "On some exact solutions of the nonlinear Schrödinger equations in three spatial dimensions," Lett. Nuovo Cim. 31, 571-576 (1981).

[8] Gagnon, L. and Winternitz, P., "Lie symmetries of a generalised non-linear Schrödinger equation: I. The symmetry group and its subgroups," J. Phys. A: Math. Gen. 21, 1493-1511 (1988).

[9] Gagnon, L. and Winternitz, P., "Lie symmetries of a generalised non-linear Schrödinger equation: II. Exact solutions," J. Phys. A: Math. Gen. 22, 469-497 (1989).

[10] Gagnon, L. and Winternitz, P., "Lie symmetries of a generalised non-linear Schrödinger equation: III. Reductions to third-order ordinary differential equations," J. Phys. A: Math. Gen. 22, 499-509 (1989).

[11] Gagnon, L. and Winternitz, P., "Exact solutions of the cubic and quintic non-linear Schrödinger equation for a cylindrical geometry," Phys. Rev. A 39, 296-306 (1989).

[12] Gagnon, L. and Wintenitz, P., "Symmetry classes of variable coefficient nonlinear Schrödinger equations," J. Phys. A: Math. Gen. 26, 7061-7076 (1993).

[13] Ivanova, N., "Symmetry of Nonlinear Schrödinger Equations with Harmonic Oscillator Type Potential," Proceedings of Institute of Mathematics of NAS of Ukraine, 43, 149-150 (2002).

[14] Lie, S., "Über die Integration durch bestimmte Integrale von einer Klasse linear partieller Differentialgleichung," Arch. for Math. 6, 328-368 (1881). (Translation by N.H. Ibragimov: Lie, S. "On integration of a Class of Linear Partial Differential Equations by Means of Definite Integrals," CRC Handbook of Lie Group Analysis of Differential Equations, Vol. 2, 473-508 1994).

[15] Miller, W. Symmetry and Separation of Variables (Reading, Addison-Wesley, 1977).

[16] Nattermann, P. and Doebner, H.-D., "Gauge classification, Lie symmetries and integrability of a family of nonlinear Schrödinger equations," J. Nonlinear Math. Phys. 3, 302-310 (1996).

[17] Niederer, U., "The maximal kinematical invariance group of the free Schrödinger equation," Helv. Phys. Acta. 45, 802-810 (1972).

[18] Niederer, U. "The maximal kinematical invariance group of the harmonic oscillator," Helv. Phys. Acta. 46, 191-200 (1973).

[19] Nikitin, A.G. and Popovych, R.O. "Group classification of nonlinear Schrödinger equations," Ukr. Math. J. 53, 1255-1265 (2001)

[20] Olver, P., Applications of Lie groups to differential equations (New-York, Springer-Verlag, 1989).

[21] Ovsiannikov, L.V., Group analysis of differential equations (New York, Academic Press, 1982).

[22] Popovych, R.O. and Ivanova, N.M., "New results on group classification of nonlinear diffusion-convection equations," math-ph/0306035, 19 p.

[23] Popovych, R.O. and Ivanova, N.M. and Eshraghi, H., "Lie Symmetries of (1+1)-Dimensional Cubic Schrödinger Equation with Potential," math-ph/0312055, 6 p.

[24] Popovych, R.O. and Yehorchenko, I.A., "Group classification of generalized eikonal equations," Ukr. Math. J. 53, 1841-1850 (2001). (See math-ph/0112055 for the extended version.)

[25] Zhdanov, R.Z. and Lahno, V.I., "Group classification of heat conductivity equations with a nonlinear source," J. Phys. A.: Math. Gen. 32, 7405-7418 (1999).

[26] Zhdanov, R. and Roman, O., "On preliminary symmetry classification of nonlinear Schrödinger equation with some applications of Doebner-Goldin models," Rep. Math. Phys. 45, 273-291 (2000). 